Session II

OBSERVATIONS 


\title{
THE ABSOLUTE SPECTROPHOTOMETRY OF 170 PLANETARY NEBULAE
}

\author{
B. A. Vorontsov-Velyaminov, E. B. Kostjakova, \\ O. D. Dokuchaeva, V.P. ARHipova
}

(Sternberg State Astronomical Institute, Moscow, U.S.S.R.)

An investigation of planetary nebulae has been underway at the Sternberg State Astronomical Institute in Moscow for several years. It was begun with the measurement of the emission-line intensities of planetary nebulae in an homogeneous system in absolute units. More than 300 long-exposure objective prism spectrograms were obtained with the 50-cm Maksutov telescope at the Crimean Station of the Institute and with the 70-cm meniscus telescope of the Abastumani Observatory. The dispersion of the spectrograms was 190 and $160 \AA / \mathrm{mm}$ at $\mathrm{H} \gamma$ respectively.

For photometric reductions, Vorontsov-Velyaminov's wide-slit method was used. The line intensities were derived from a comparison with the spectra of A0-type stars on the same plates. An 'average A0 V star' was adopted as a standard comparison source. Its energy distribution was computed from recently published photoelectric and photographic observations. Planetary nebulae with angular dimensions less than

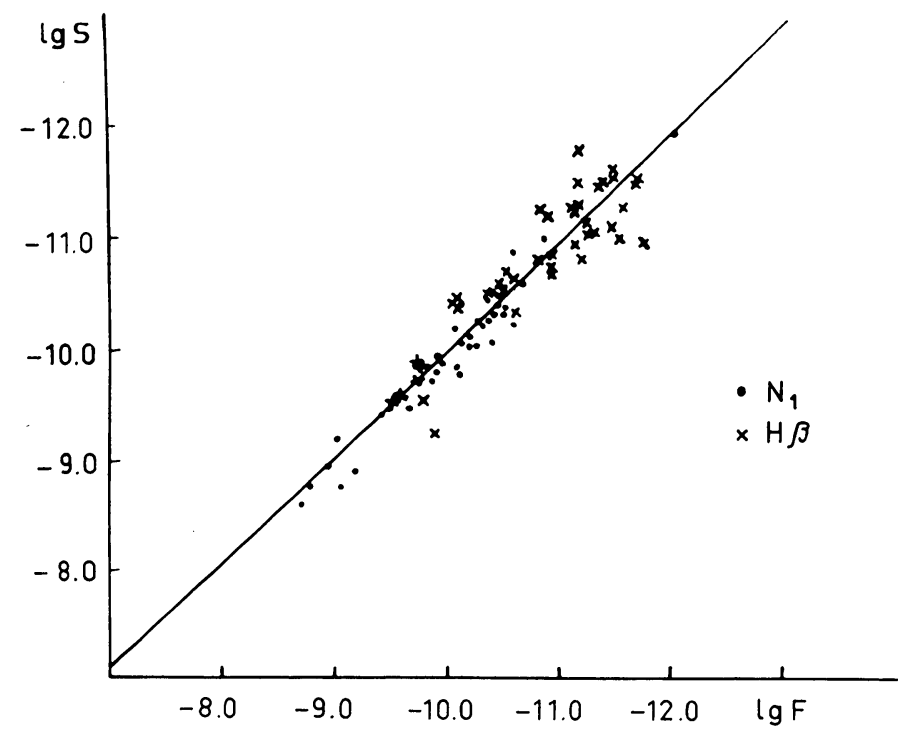

FIG. 1. Comparison of Sternberg $(\lg S)$ measurements of planetary nebulae with O'Dell's photoelectric measurements $(\lg F)$.

Osterbrock and O'Dell (eds.), Planetary Nebulae, 57-58. I.A.U. 
30 " of arc, and with total magnitudes brighter than $m=13$ were studied. The number of lines measured for the nebulae varied from 1 to 20 (from 10 to 20 lines for most of the objects) in the spectral interval 3700-6600 $\AA$. Comparison of the results showed good agreement with the data obtained by the photoelectric method, as can be seen in Figure 1 , where our $\mathrm{N}_{1}$ and $\mathrm{H} \beta$ energy fluxes are plotted against those obtained by O'Dell. The continuous spectrum intensity of the planetary-nebula nucleus was also estimated if possible. For 108 planetary nebulae the absolute line intensities were determined for the first time, while for 93 objects no data at all on the line intensities had been previously published.

The most interesting group of nearly 90 planetary nebulae is situated in the region projected on the galactic centre. These nebulae have total magnitudes of about $m=13$. The greatest majority of them have not previously been investigated by the spectroscopic method. The connection between the apparent distribution of the nebulae projected on the galactic centre region and the interstellar light absorption in this direction was also studied. It was found that these nebulae are situated farther than 2 or $3 \mathrm{kpc}$ from the Sun and that they do not form a compact group in space.

The ratio of the $\mathrm{H} \alpha$ and $\mathrm{N}_{1}$ line intensities of the nebulae in the galactic-centre region was found to exceed systematically the ratio for nebulae in other parts of the sky. This seems to indicate the possibility of a physical difference between the nebulae in the direction of the galactic centre and the nebulae situated in the other regions of the sky.

The results of the work are published in seven papers listed below, in the References.

\section{References}

Vorontsov-Velyaminov, B. A., Kostjakova, E. B., Dokuchaeva, O.D., Arhipova, V.P.(1964) Astr. Cirk., 305.

Vorontsov-Velyaminov, B. A., Kostjakova, E. B., Dokuchaeva, O.D., Arhipova, V.P. (1964) Astr. $\mathrm{Zu}, \mathbf{4 1}, 255$.

Vorontsov-Velyaminov, B. A., Kostjakova, E. B., Dokuchaeva, O.D., Arhipova, V.P.(1965) Astr. Cirk., 348.

Vorontsov-Velyaminov, B. A., Kostjakova, E. B., Dokuchaeva, O.D., Arhipova, V.P.(1965) Astr. $Z u$., 42, 464.

Vorontsov-Velyaminov, B. A., Kostjakova, E. B., Dokuchaeva, O.D., Arhipova, V.P. (1965) Astr. $\mathrm{Zu}, \mathbf{4 2}, 730$.

Vorontsov-Velyaminov, B. A., Kostjakova, E. B., Dokuchaeva, O.D., Arhipova, V.P.(1967) Astr. Cirk., 437.

Vorontsov-Velyaminov, B. A., Kostjakova, E. B., Dokuchaeva, O.D., Arhipova, V.P. (1967) Astr. $Z u ., 44,361$.

\section{DISCUSSION}

Aller: To what extent do overlapping spectra of background stars complicate the measurements of nebular line intensities?

Dokuchaeva: We used only the best spectrograms in which there was no overlapping by background stars. 